\title{
Scenic area capacity calculation method based on dynamic network analysis: the case of Mountain Lu, Jiangxi Province
}

\author{
Li Ping
}

Mathematical \& Physical College, Huanggang normal university, Huanggang, 438000, China

email: 258803198@qq.com,

Keywords: Dynamic analysis; environmental capacity; visitor flow

Abstract. Many managers and researchers would agree that, accompanying with uncertainty and dynamism, capacity of a scenic area is hard to compute usually. In order to fulfill this purpose, we construct an estimating method based on dynamic network analysis, and verify the validity of the model by a case study of Mountain Lu.

\section{Introduction}

In order to maintain natural environment and ecological quality, it is necessary to determine the capacity of scenic area. Capacity measurement of natural environment is a complicated problem, because it need consider the following factors: economy, ecology, pollution, etc.

A large amount of research concerned this topic. Yanhuili and Luojing[1] developed a model based on production line. Duyao and Puyun[2] discussed the method of natural capacity by discrete computing. Pengyu[3] established a supply chain with equilibrium management tool.

In this paper, a novel method based on dynamic network analysis is put forward. In this model, tourist facilities including scenic spot, restaurant, hotel etc. are simplified as joints, the road connect with them are simplified as bi-directional routes. Joints and bi-directional routes constitute an idealized network, and the problem of environmental capacity is simplified as a calculating of network's tolerance.

\section{Calculation of capacity based on network analysis}

Consider with the requirement of tourists, there are different types of facilities need be built in scenic area. Such as entrance of scenic area, recreation facilities, restaurant, hotel, bus stations, toilet and etc. Some of them are essentials for a tourist activity, when others are selectable. The former include entrance of scenic area, restaurant, hotel and bus station, the latter include optional recreation facilities and concrete scenic spot.

On the premise of idealized, a scenic area can be mapped as a network including joints and routes. Joints represent destinations of tourists while routes represent the directions of movement. Joints in this network consist of two types: key joints and normal ones. Key joints represent essential destinations and normal ones represent selectable destinations. Figure 1 below will give a sign of relationship between them.

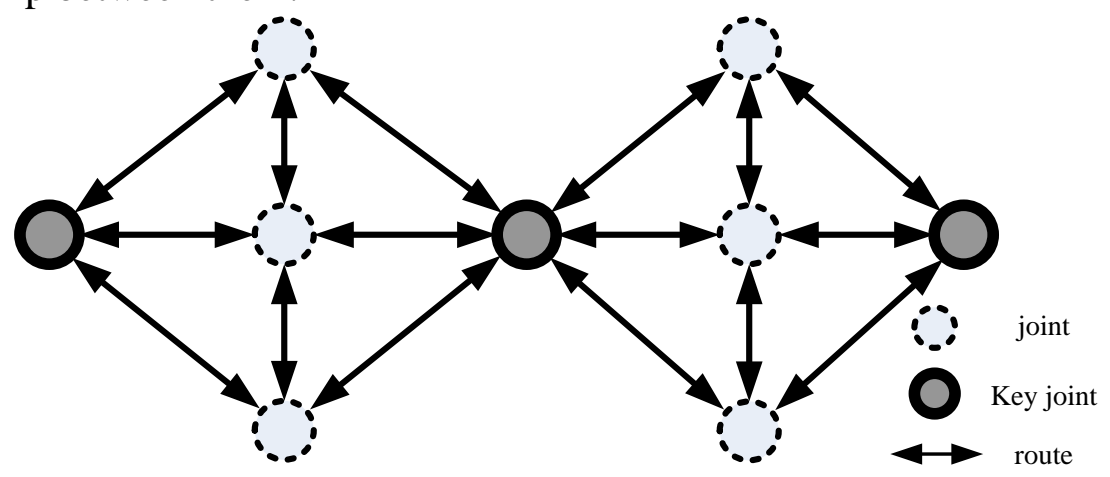

Fig.1. Network shape 
From the perspective of computational complexity,following feature parameters will be accepted, in those formula, $|E|$ means the number of routes in the network, $J$ means joint of the network, and $r_{j k}$ represent the route between the joint $j$ and the joint $k$ :

Network complexity(NC):

$N C=\frac{|E|-(J-1)}{\frac{J^{2}}{4}-(J-1)}=\frac{4|E|-4 J+4}{(J-2)^{2}}$

Resource factor(RF):

$R F=\frac{1}{J} \frac{1}{K} \sum_{j=1}^{J} \sum_{k=1}^{K} \operatorname{sgn}\left(r_{j k}\right)$

Where $\operatorname{sgn}(\cdot)$ represent the sign function:

$\operatorname{sgn}\left(r_{j k}\right)=\left\{\begin{array}{cc}1 & \text { if } r_{j k}>0 \\ 0 & \text { else }\end{array}\right.$

Resource strength(RS):

$R S_{k}=\frac{R_{k}}{\frac{1}{J} \sum_{j=1}^{J} r_{j k}}$

Resource constrainedness(RC):

$$
R C_{k}=\frac{\sum_{j=1}^{J} r_{j k}}{R_{k} \sum_{j=1}^{J} \operatorname{sgn}\left(r_{j k}\right)}
$$

Considering the capacity constraints of scenic area in rush hour, there will be a large number of tourists waiting at the key joint, such as the entrance of the scenic area, restaurant, bus station, etc. In the most simplified case, normal joints are supposed to have Unlimited supply capacity. So, the capacity of whole scenic area is depend on the supply capacity of key joint.

\section{Case study}

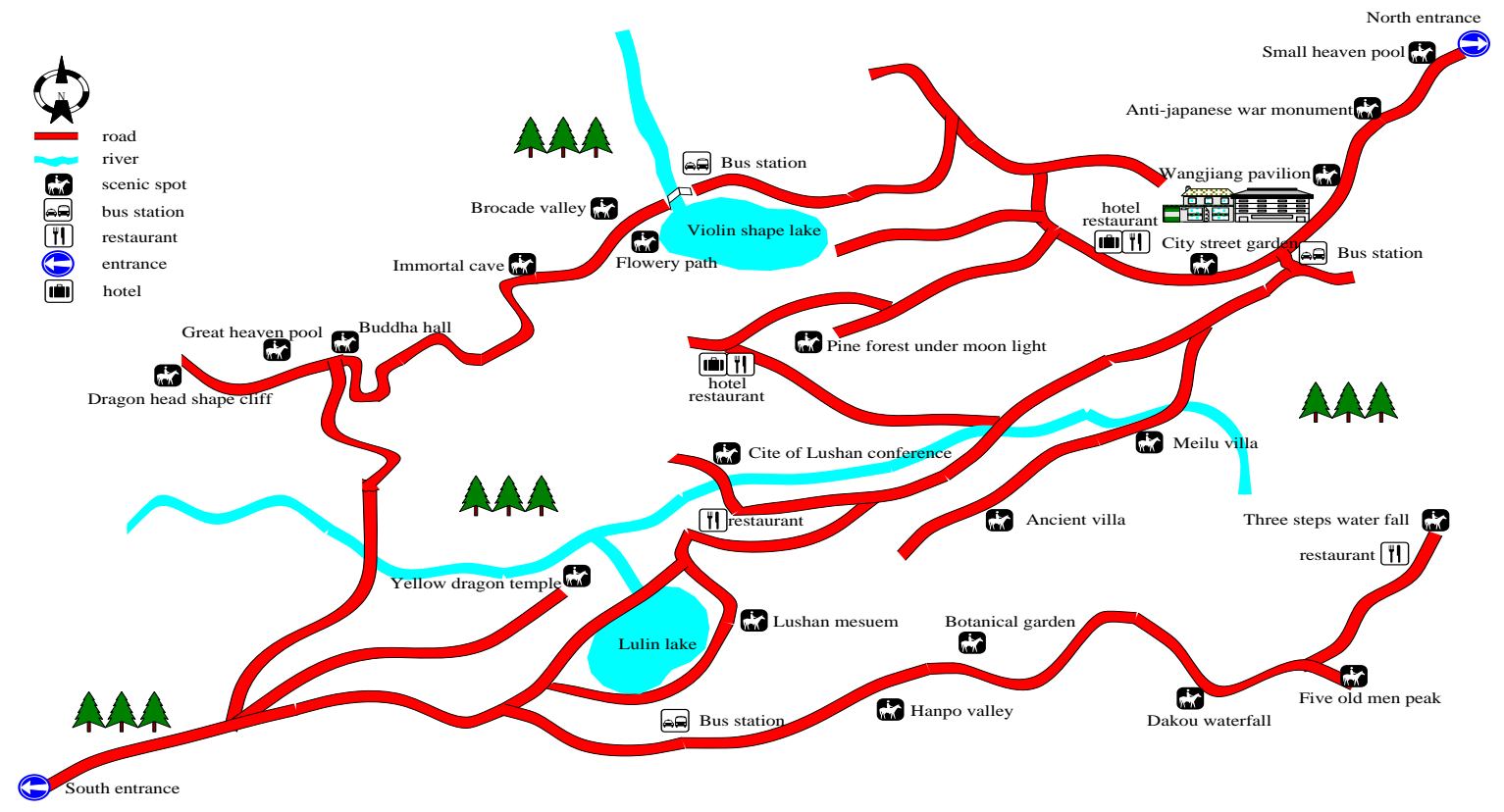

Fig.2. Sketch map of Mountain Lu 
In this paper, Mountain Lushan scenic area is considered as the object of case study. Mountain Lushan is located in the northern part of Jiangxi Province, to the south of the Yangtze River and northwest of the Poyang Lake. According to records, the scenic area, covering 302 square kilometers, houses 16 natural wonders, 474 scenic spots and 171 peaks, of which the highest peak, the Dahanyang Peak, is 1,474 meters above sea level.

According to the statistical data, the maximum flow of visitors had gone over 50,000 in some national holidays. The three most important factors restrict the development of Mountain Lushan include public traffic, catering and accommodation. In order to optimize the capability of receiving visitors, the bottleneck joints should be found out and improved. Figure 2 will give a sketch map of Mountain Lushan.

By simplifying the facilities and scenic spots into joints, the sketch map is converted as network in following Figure 3.

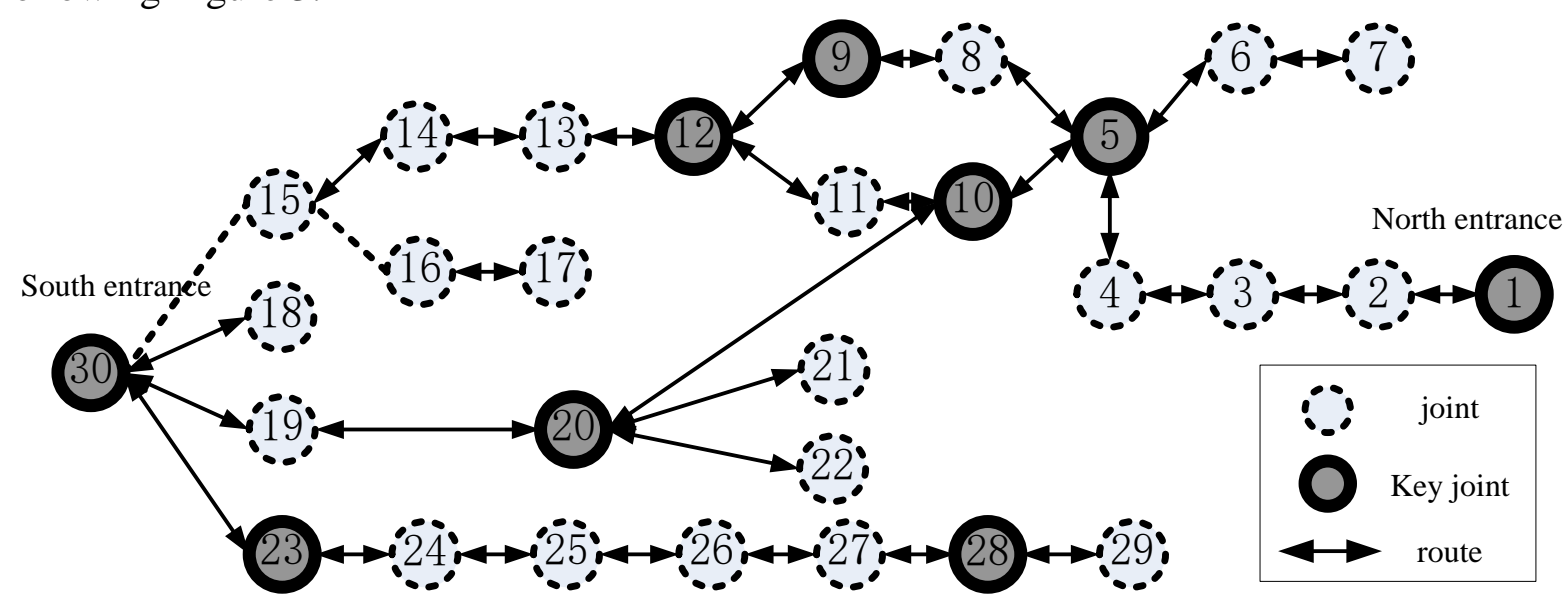

Fig.3. Network of Mountain Lu

By computing the Resource constrainedness(RC) of nine key joints, the sequence of them can be obtained as follow(from large to small):

(30) $>(1)>($ (5) $>(12)>(20)>(10)>(9)>(23)>(28)$

Because the joint (30) and (1) represent the entrances of scenic area, whose capacity supposed are unlimited, so the three most important joints need be improved are (5), (12) and (20). In the actual situation, corresponding bus stations and restaurants are the biggest concerns in the scenic area.

\section{Conclusion}

The paper analysis some type of factors that influence the capacity of scenic area, and convert the map of scenic area into a kind of simplified network consist of joints and routes. By computing the parameters, the most urgency joints can be found and used as the direction of improving the capacity of scenic area.

\section{References}

[1] Yanhuili, Luojing: A new calculation method of tourism space carrying capacity based on production line. Human Geography, vol. 125 (2012), p. 115-117, 155.

[2] Duyao, Puyun: Calculation method and application of naturally environmental capacity of scenic area based on dynamic discrete analysis. Market modernization, vol. 529 (2008), p. 335.

[3] Pengyu: Equilibrium management of scenic area capacity---case study of jiuzhaigou valley. Journal of Chongqing city management vocational college, vol. 9 (2009), p. 17-20.

[4] Shouyongyi: Resource-constrained multi-project scheduling models and methods. Zhejiang university press, Hangzhou, 2010. 
[5] Zhaoming, Zhaopeng, Lixiujuan: Calculation methods of environmental capacity and tourist capacity ---case study of Mountain wuling ecotourism area. Hebei forestry science and technology, vol. 2 (2014), p. 40-41.

[6] Yanhuili, Xionghao: The Access Batch and the Maximum Carrying Capacity of Scenic. Tourism Tribune, vol. 28 (2013), p. 83-87. 